\title{
A Miniaturized High-Gain Flexible Antenna for UAV Applications
}

\author{
Xinhuan Yang, Yanzhu Qi, Bo Yuan, Yazi Cao $\mathbb{D}$, and Gaofeng Wang $\mathbb{D}$ \\ Hangzhou Dianzi University, Hangzhou, Zhejiang, China \\ Correspondence should be addressed to Yazi Cao; yazi.cao@hotmail.com and Gaofeng Wang; gaofeng@hdu.edu.cn
}

Received 3 April 2021; Accepted 8 July 2021; Published 20 July 2021

Academic Editor: Giovanni Andrea Casula

Copyright (C) 2021 Xinhuan Yang et al. This is an open access article distributed under the Creative Commons Attribution License, which permits unrestricted use, distribution, and reproduction in any medium, provided the original work is properly cited.

A miniaturized high-gain flexible unmanned aerial vehicle (UAV) antenna is presented in this study. The proposed antenna basically comprised of three parts of printed patch in series, etched on dielectric substrate. And, a flexible cable is loaded on the bottom of dielectric substrate. A coplanar waveguide (CPW) with asymmetric ground feeding structure is employed to provide good impedance matching. The surface current can achieve the same phase for the straight-line patch and the flexible cable, through adjusting the dimensions of the meander line patch, which increases radiation gain while maintaining the compact size. As an important merit to be highlighted, the flexible cable can greatly reduce the volume and aerodynamic drag of the antenna. It has a low-profile compact size of $196 \times 15 \times 0.8 \mathrm{~mm}^{3}$ (excluding flexible cable). The results show that the omnidirectional gain fluctuates within $4.5 \pm 0.1 \mathrm{dBi}$ in the desired band $(902 \mathrm{MHz}-928 \mathrm{MHz})$, which is high enough for the UAV application. Details of the antenna design and experimental results are presented and discussed.

\section{Introduction}

In the recent years, the applications of unmanned aerial vehicles (UAVs) have attracted lots of attention in the area of communication, military, and commercial market. They are widely used for exploration, reconnoiter, and multimedia communication. It is necessary to have reliable communication link between ground control unit and an aircraft for transmitting telemetry. Unfortunately, there are some restrictions for UAVs' antennas. Firstly, the fly time of drone is quite limited due to the lithium battery life. Therefore, the larger the physical volume of antennas, the huger the space occupied on the UAVs, the greater the wind drag, and the shorter the fly time of UAVs. Secondly, the ratio of flight distance and height is large for the UAVs, which determines that the radiation pattern of the antenna must be omnidirectional in the horizontal plane [1-5]. In view of these factors, antennas are required to have small effective area, slight weight, and omnidirectional radiation.

In typical applications, the images and data transmission antennas of UAVs need to meet transmission distance more than 10 kilometer, whereas the data transmission power is 1 watt and its reception power is 500 milliwatts. Nowadays, high-quality and high-speed transmission of data and images' systems with compactness is extremely important in UAVs' applications [6]. For improving the radiation characteristics, some metamaterial circularly polarized antennas with various geometries are presented in [7-9], which have high gain and compact size. However, low costs, vertical polarization, and omnidirectional radiation coverage of monopoles in the azimuth plane make them very attractive for UAV applications. So, it is worth trying to combine the metamaterials with monopole antennas. And, a variety of antennas have been used on the UAVs to achieve the required radiation pattern. The authors of [10-15] designed antennas that can achieve the omnidirectional radiation pattern by using printed monopole or dipole. A horizontally polarized omnidirectional loop antenna using the segmented line was proposed in [16]; over the frequency range of $2.35-2.55 \mathrm{GHz}$, the reflection coefficient is less than $-10 \mathrm{~dB}$, but the maximum gain is only up to $2 \mathrm{dBi}$. As reported in [17], although segment loop antenna has compact size and omnidirectional radiation at $956 \mathrm{MHz}$, it has low gain. Weiyu et al. [18] designed a dual band and low-profile antenna with omnidirectional pattern in the horizontal plane. In [19], a $0.5-2 \mathrm{GHz}$ (VSWR <3:1) blade monopole antenna was presented. However, this kind of antenna needs a huge ground plane, leading to a large overall size. Roughly 
speaking, monopole or dipole antennas have been widely used in wireless communication systems because of simple structures, low costs, and omnidirectional radiation pattern, which also offer advantages in UAVs' applications. However, most of them hardly achieve the small dimensions at low frequencies, which make them difficult for integration in UAVs. To improve the performance of UAVs, the miniaturized monopole antennas with high gains are highly desirable.

In this work, a miniaturized high-gain flexible monopole antenna for UAVs' application is presented. The radiating section, formed by a straight-line patch, a meanderline patch and a flexible cable, and a compact feeding network, is designed carefully to provide high-gain omnidirectional radiation. The novelty of this design is that a flexible cable, which has small cross-sectional area and low-loss material, replaces part of the radiating patch, therefore reducing aerodynamic drag. The use of low-loss, flexible, and lightweight design is also an advantage of this antenna. As an important advantage to be highlighted, its maximum gain is up to $4.56 \mathrm{dBi}$, which is about $2.2 \mathrm{dBi}$ higher than traditional dipole antennas, and the flying distance can be achieved about 1.2 times longer.

\section{Antenna Design and Analysis}

As the successive half wavelength current maxima are in the opposite phase and if the currents were equal, there would be perfect cancellation of the radiation from the oppositely phased pairs of current maxima. However, if all the current maxima were in phase, the radiated fields would add, and a high gain could be achieved. The way of achieving this phase reversal is by inserting an antiresonant network, which uses radiating elements that are a little longer or shorter than one half wavelength. The self-reactance of the longer or shorter dipole is then used in the design of the phasing network between the elements to achieve the desired overall phase shift. In this design, a meander line patch is used to as an antiresonant network, which makes the same phase for the straight-line patch and the flexible cable so that the antenna achieves high-gain radiation.

The geometry of the proposed UAV antenna with detailed dimensions is shown in Figure 1. The various configuration parameters of antenna are as follows: $W=15 \mathrm{~mm}, \quad w_{1}=0.9 \mathrm{~mm}, \quad L=196 \mathrm{~mm}, \quad l_{1}=41.5 \mathrm{~mm}$, $l_{2}=83 \mathrm{~mm}, l_{3}=1 \mathrm{~mm}, l_{4}=1.5 \mathrm{~mm}, l_{5}=9.5 \mathrm{~mm}, l_{6}=153 \mathrm{~mm}$, $a=27 \mathrm{~mm}, b=5 \mathrm{~mm}, c=1 \mathrm{~mm}, d_{1}=0.95 \mathrm{~mm}, d_{2}=2 \mathrm{~mm}$, $d_{3}=1 \mathrm{~mm}$, and $d_{4}=1.3 \mathrm{~mm}$. The diameter of the flexible cable is $2 \mathrm{~mm}$, whose material is stainless steel. For versatility, an FR-4 substrate (thickness $=0.8 \mathrm{~mm}$ and $\varepsilon_{r}=4.4$ ) is adopted.

As seen, the antenna is mainly made up of three parts' patches and a flexible cable (e.g., Part 1, Part 2, and Part 3). Among them, the coplanar waveguide (CPW) feeding structure is displayed in Part 1, comprised of a pair of asymmetric rectangular patches. It is employed to provide good impedance matching, even though it has no radiated effect as a transmission line. A straight-line patch and a meander line, used as part 2 and part 3, respectively, are located on the top of the dielectric substrate. On the bottom of the dielectric substrate, a groove whose two sides are coated with metal is equipped with a flexible cable.

Compared to the microstrip line, the coplanar waveguide (CPW) has lower loss and is more convenient for series connections on the same side of the substrate without via holes. Therefore, the overall design adopts asymmetric CPW structure for feeding and forms stepped slots and rectangular slots for coupling, which can improve the impedance matching between the feeding port and antenna. The highfrequency structure simulator (HFSS) is used to simulate the design. A prototype of the proposed UAV antenna is fabricated and assembled.

Particularly, Figure 1(f) shows that the flexible cable can be bent when the drone lands on the ground, whereas restored to its original shape when taking off. As a consequence, the flexible cable is adopted as the end of the antenna, resulting in little air resistance to the drone, compared with the radiated patch printed on the dielectric substrate.

Figure 2 illustrates that the impedance and bandwidth of the proposed antenna can be adjusted by changing the size of the straight-line patch, whereas the resonant frequency of the proposed antenna can be adjusted by changing the dimensions of the meander line. Using $W$ as an example, when its value is $1 \mathrm{~mm}$, the performance of $S_{11}$ is not ideal. It has a slight offset towards the low end, but keeping wide impedance bandwidth. The performance of $S_{11}$ is the best when its value is $3 \mathrm{~mm}$. However, its impedance bandwidth is very narrow. As the value increases, the impedance bandwidth of the antenna increases gradually. From these plots, it can be observed that the value of $W$ has a large influence on the reflection coefficient.

Likewise, when the length of the meaner-line patch is small, the resonant frequency is towards slightly the high end. As the value of $d_{2}$ increases, the resonant frequency of the antenna moves gradually to the low frequency, but its impedance bandwidth and the performance of $S_{11}$ are virtually unchanged. It can be seen that the value of $d_{2}$ has a large influence on the resonant frequency. A set of optimal values can be obtained by optimization and fine tuning.

The simulated current distributions at $915 \mathrm{MHz}$ are shown in Figure 3. Due to the effect of the meander line patch, there are two current zero points. Consequently, the surface currents of the straight-line patch and the flexible cable are in the same phase, which can enhance greatly the antenna omnidirectional gain. With the varying length of the meander line patch, the positions of the current zero points can be changed. When the value of $d_{2}$ is $1 \mathrm{~mm}$, one current zero point appears at the straight-line patch near the feed port, while another one appears at the meander line close to the flexible cable. As the value increases, the current zero point at the straight-line patch moves gradually to the meaner line, whereas another one moves gradually to the flexible cable. From the measured results, the proposed antenna can achieve the optimal performance when the value of $d_{2}$ is $2 \mathrm{~mm}$. 


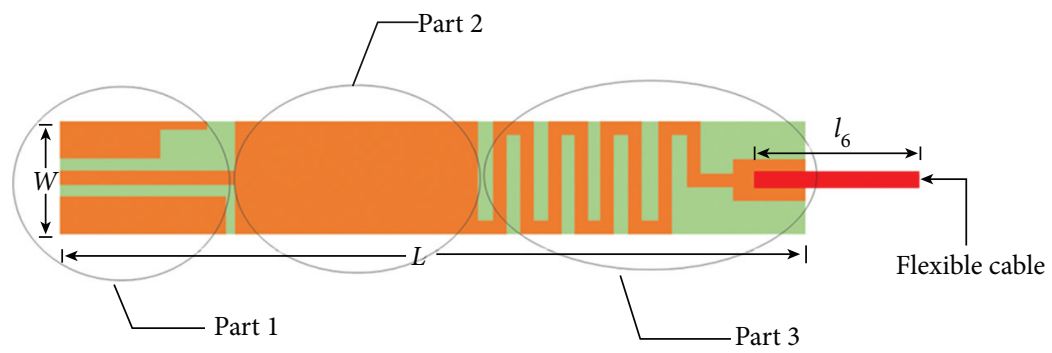

(a)

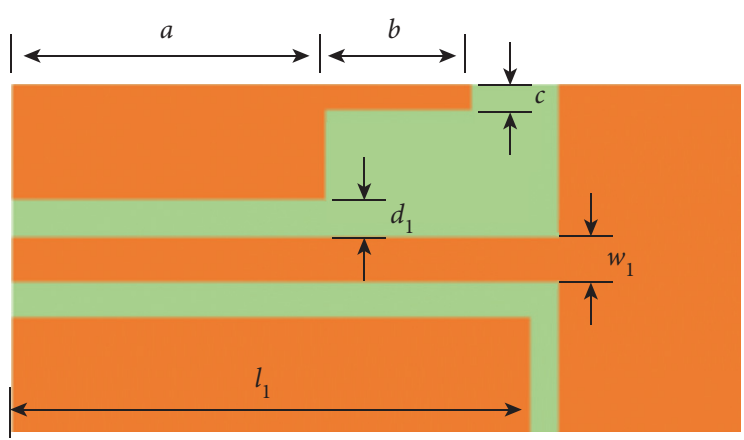

(b)

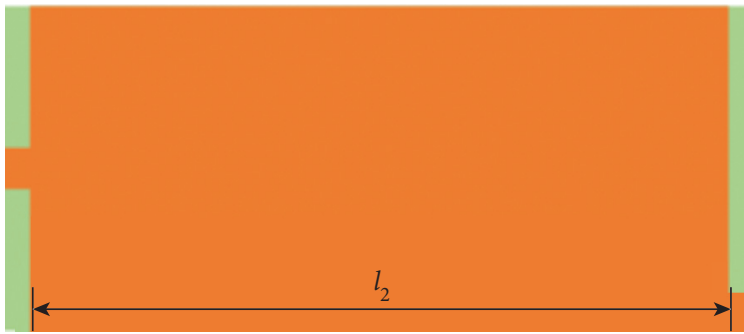

(c)

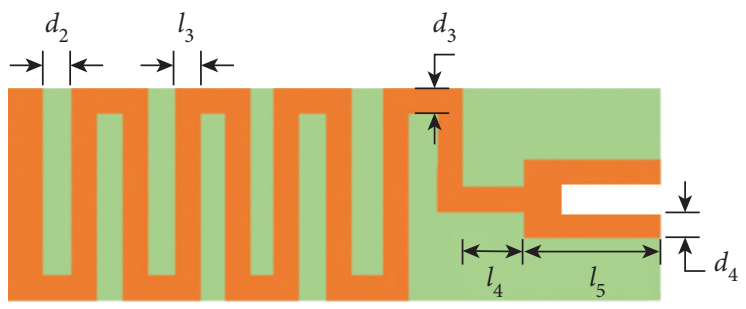

(d)

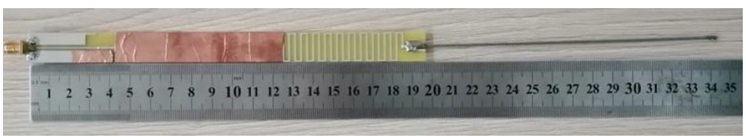

(e)

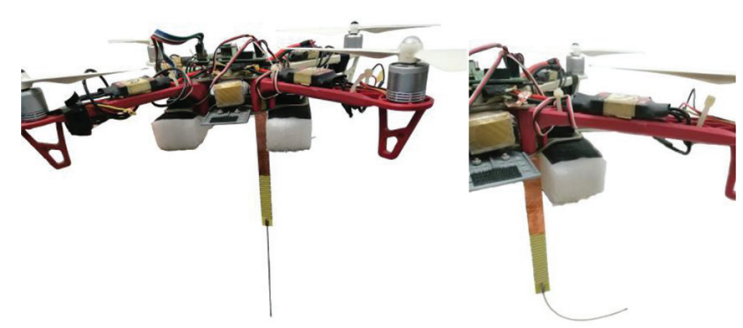

(f)

Figure 1: Geometry of the proposed antenna. (a-e) Detailed dimensions of the antenna (top view). (b) Part 1, (c) part 2, (d) part 3, and (f) the flexibility of antenna display. 


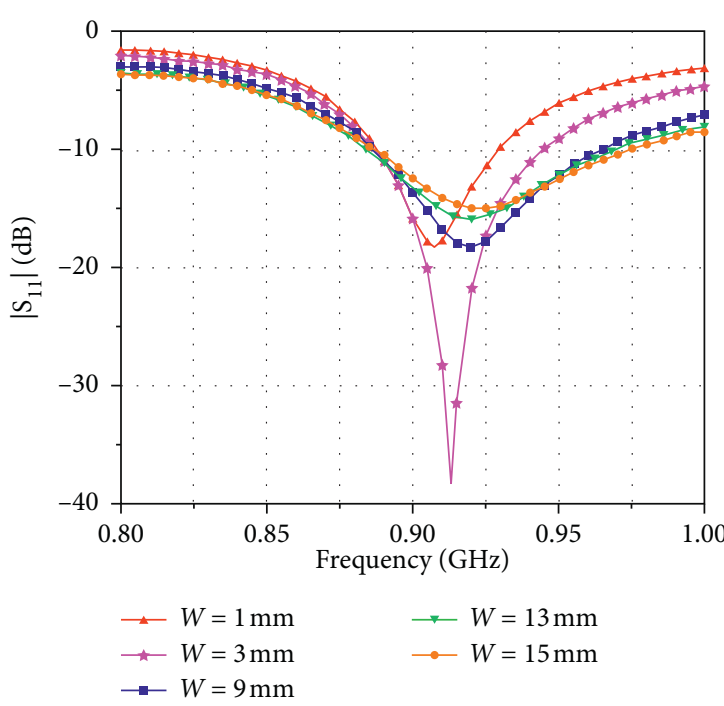

(a)

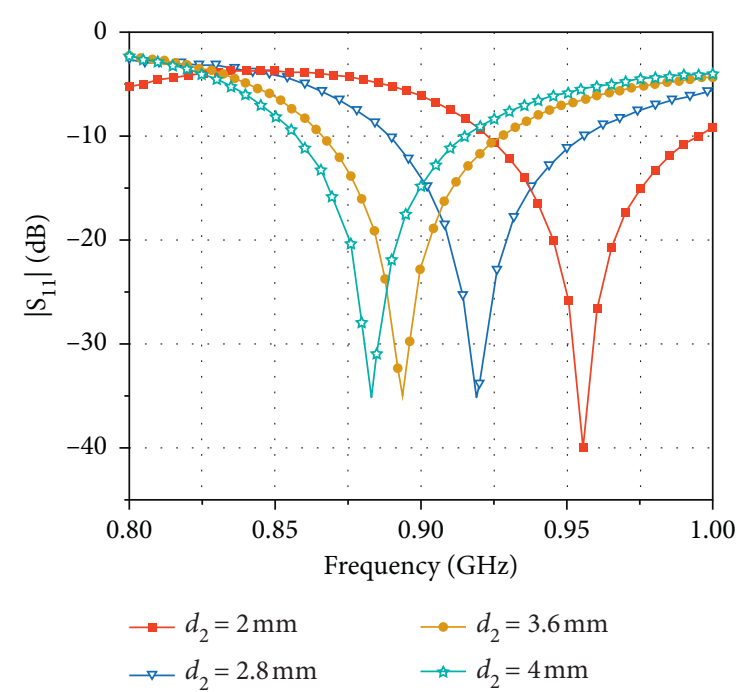

(b)

FIgURE 2: Simulated $\left|S_{11}\right|$ of the proposed antenna with variations of values: (a)W and (b) $d_{2}$.

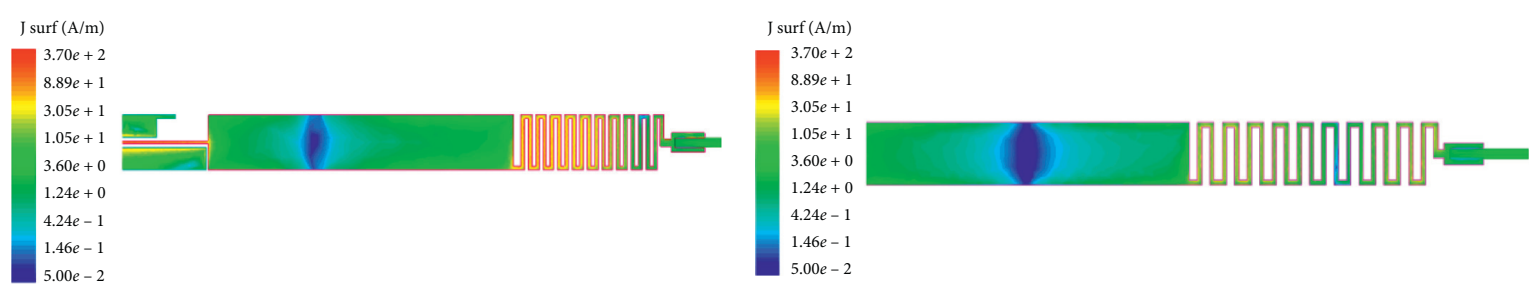

(a)

(b)

(c)

FIgURE 3: Simulated current distributions at various lengths of the meander line: (a) $d_{2}=1 \mathrm{~mm}$, (b) $d_{2}=2 \mathrm{~mm}$, and (c) $d_{2}=3 \mathrm{~mm}$.

\section{Result and Discussion}

As shown in Figure 4, the performance of antenna can be still optimal when the flexible cable is bent. It can operate well even if there is a slight frequency shift. Moreover, the performance restores immediately to the optimal when it restores to its original shape, thereby reducing effectively the volume of antenna yet guaranteeing the good performance.

Figure 5 depicts the measured and simulated $S_{11}$ of the proposed UAV antenna with the dimensions given in Figure 1. Because a little copper was added on the substrate for debugging during the test, the measured results are better than the simulated ones. It can achieve VSWR $<1.22$ : $1\left(\left|S_{11}\right|<-20 \mathrm{~dB}\right)$ at the whole operating bandwidth $902 \mathrm{MHZ} \sim 928 \mathrm{MHz}$.

The measured and simulated gain of the proposed UAV antenna is shown in Figure 6. Because the actual loss is larger than the ideal situation and the measurement error, the measured gain is slightly lower than the simulated gain of the UAV antenna. The result shows that proposed antenna achieves the high gain of $4.56 \mathrm{dBi}$ in the $902 \mathrm{MHz} \sim 928 \mathrm{MHz}$. This gain of the proposed antenna can be accepted in practical UAVs applications. The measured radiation efficiency of the proposed UAV antenna are shown in Figure 7, and the result shows that the proposed antenna achieves the high radiation efficiency in the $902 \mathrm{MHz} \sim 928 \mathrm{MHz}$.

The test environment of antenna is shown in Figure 8. The radiation patterns of the fabricated prototype at $915 \mathrm{MHz}$ are shown in Figure 9, in which an omnidirectional radiation pattern with linear polarization can be observed. Although the size of antenna is relatively small, the antenna gain is rather high. It shows that this antenna has good omnidirectional radiation characteristics. Therefore, the 


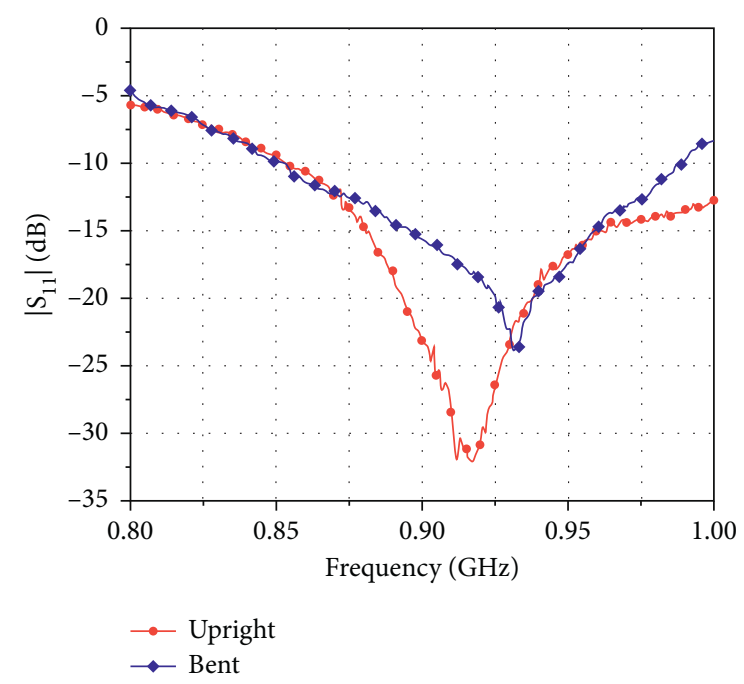

FIgURE 4: Influence of the flexible cable on $\left|S_{11}\right|$ for the proposed antenna.

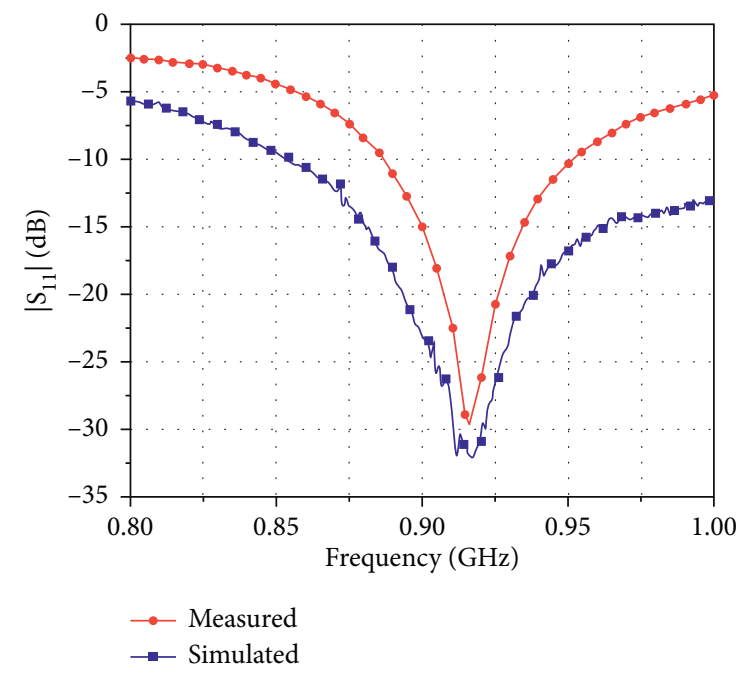

FIgURE 5: Measured and simulated $\left|S_{11}\right|$ for the proposed antenna.

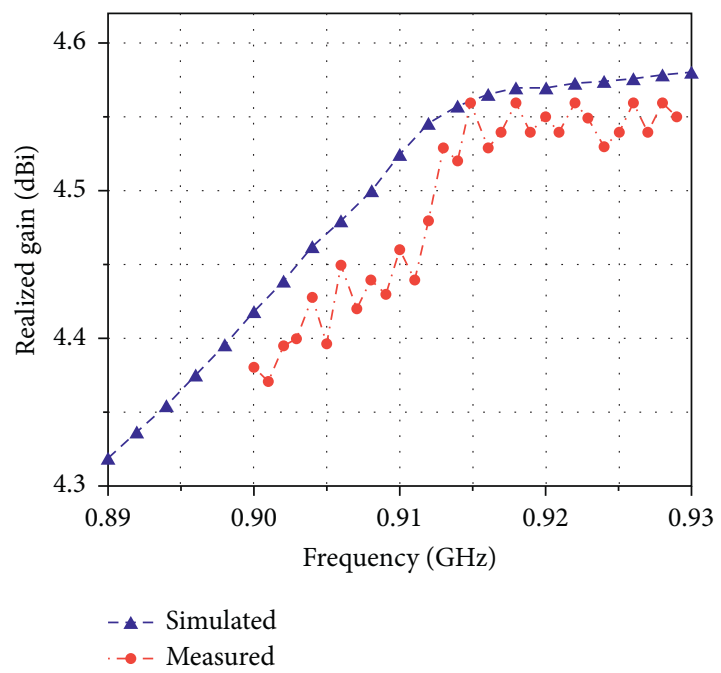

FIgURE 6: Measured and simulated gain of the antenna.

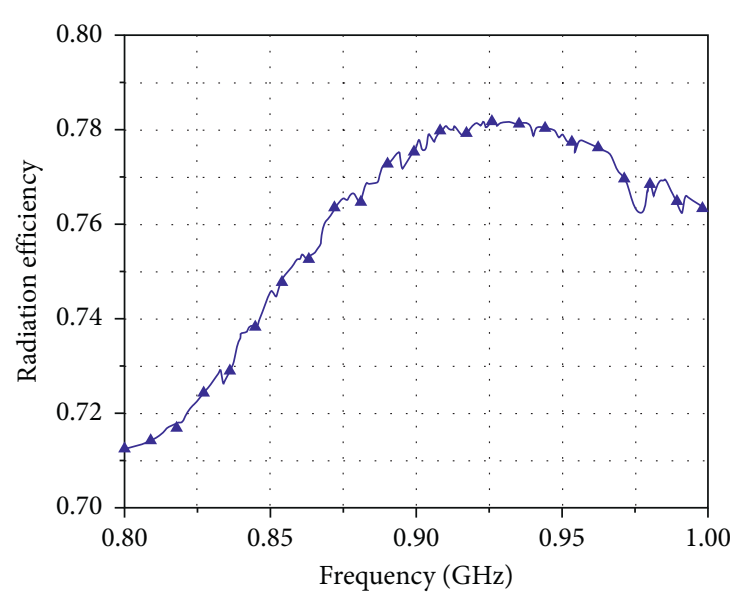

Figure 7: Measured radiation efficiency of the antenna.

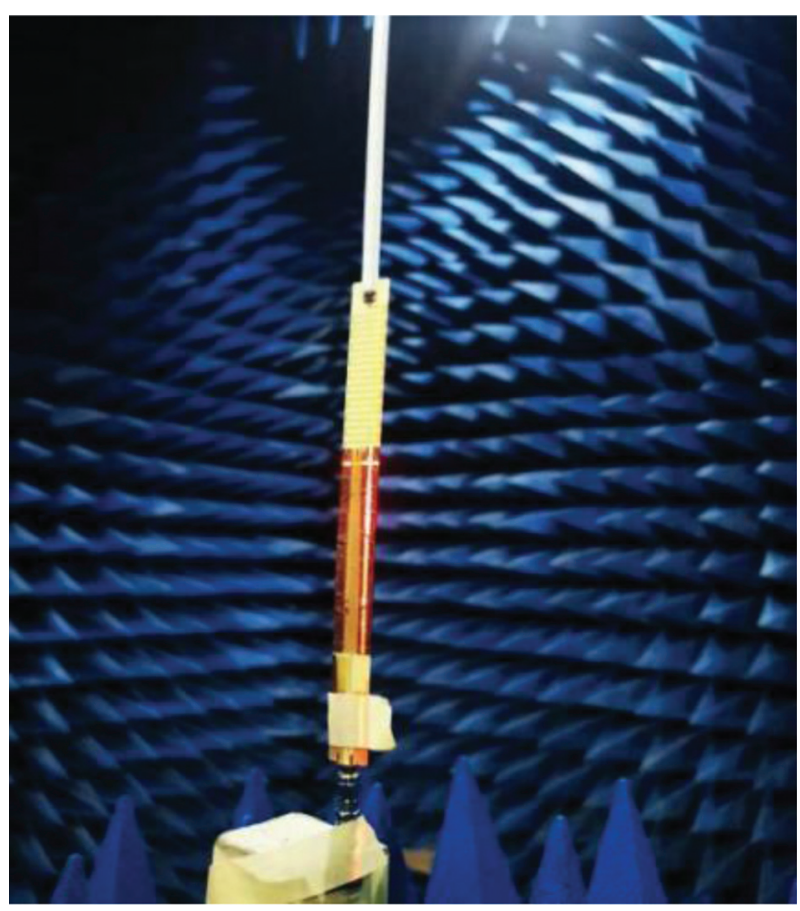

FIgURE 8: The environment of measured UAV antenna.

antenna in the drone can receive signals from any angle on the horizontal plane because of omnidirectional radiation in the horizontal plane.

The performance comparison of the proposed antenna to publish referenced works is made in Table 1 . It uses the wavelength to measure the size of the PCB. The miniaturization of the antenna is reflected in the use of flexible cables instead of radiating patches as part of the radiation, thereby reducing the length of the dielectric substrate. From Table 1, it could be seen that the proposed antenna provides the higher gain with a relatively reduced size. Furthermore, it has a relatively small size. It could be concluded that the proposed antenna with a reduced size has a good radiation properties and a simple feeding network. 


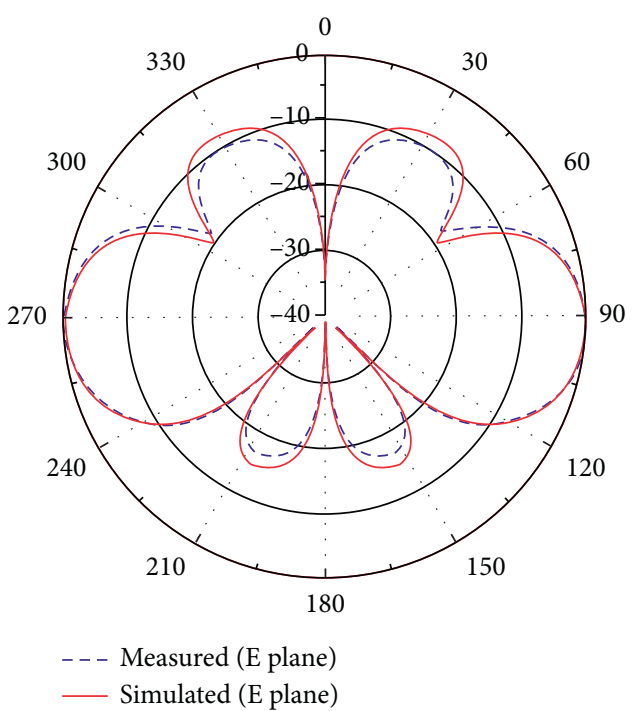

(a)

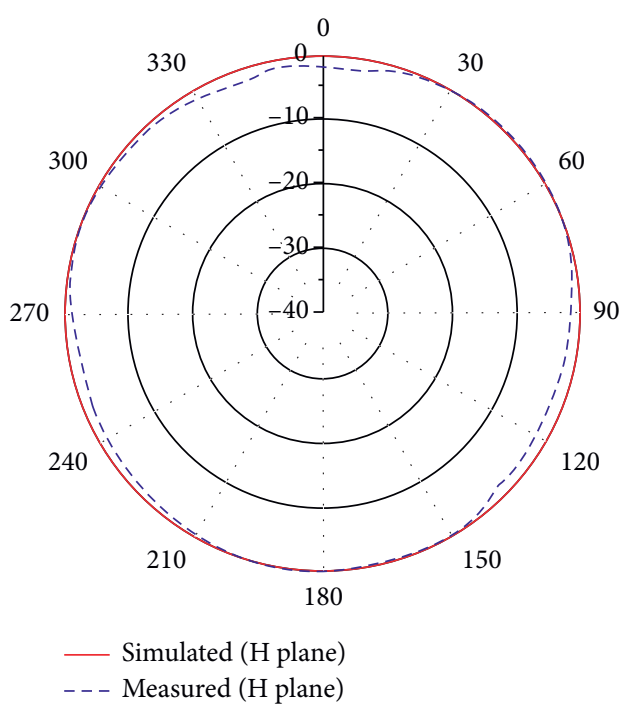

(b)

FIgURE 9: Measured and simulated radiation patterns at (a) E plane and (b) $\mathrm{H}$ plane.

TABLE 1: Comparison of other omnidirectional antennas and this work.

\begin{tabular}{lccccc}
\hline Ref. & Center frequency $(\mathrm{GHz})$ & The size of PCB $\left(\lambda_{0}\right)$ & Antenna substrate height $(\mathrm{mm})$ & Substrate $\varepsilon_{r}$ & Realized gain variation $(\mathrm{dBi})$ \\
\hline$[5]$ & 0.868 & $0.69 \times 0.2$ & 0.127 & 2.17 & $\sim 2.6$ \\
{$[9]$} & 5.2 & $0.366 \times 0.366$ & 2.4 & 4.4 & $\sim 4.17$ \\
{$[10]$} & 5.3 & $0.54 \times 0.54$ & 0.8 & 4.4 & $\sim 3.4$ \\
{$[12]$} & 6.2 & $1.5 \times 0.28$ & 1.6 & 4.4 & $\sim 4.1$ \\
{$[17]$} & 2.45 & $0.41 \times 0.41$ & 0.5 & 4.4 & $\sim 2$ \\
{$[19]$} & 1.25 & $0.4 \times 0.25$ & 1.6 & 4.4 & $\sim 4.56$ \\
This work & 0.915 & $0.6 \times 0.046$ & 0.8 & \\
\hline
\end{tabular}

\section{Conclusion}

In this work, a miniaturized high-gain flexible UAV antenna, which consists of a straight-line patch, a meander line, and a flexible cable printed on a dielectric substrate, has been proposed, fabricated, and tested. The flexible cable can be bent when the drone lands on the ground, whereas be straight when taking off. As a consequence, it can reduce greatly the dimensions of the whole antenna without compromising the radiating performance. It is found that, by adjusting the dimensions of the straight-line patch, the impedance and bandwidth of the antenna can be controlled effectively.

By adjusting the dimensions of the meander line patch, the surface current can be made in the same phase at both the straight-line patch and the flexible cable, which is very helpful to generate an omnidirectional radiation with a highgain level in the operating frequency range. The proposed antenna can cover $902 \mathrm{MHz} \sim 928 \mathrm{MHz}$ with VSWR $<1.22$ : 1. Moreover, the antenna has a low-profile structure of $341 \times 15 \times 0.8 \mathrm{~mm}^{3}$. Its weight is about $15 \mathrm{~g}$. The proposed antenna is very suitable for UAVs applications.

\section{Data Availability}

The data used to support the findings of the study are available from the corresponding author upon request.

\section{Conflicts of Interest}

The authors declare that they have no conflicts of interest.

\section{Acknowledgments}

This work was supported by the National Key Research and Development Program of China, under Grant no. 2019YFB2205003.

\section{References}

[1] Y. Chen and C.-F. Wang, "Electrically small UAV antenna design using characteristic modes," IEEE Transactions on Antennas and Propagation, vol. 62, no. 2, pp. 535-545, 2014.

[2] F. Trotta, A. Manna, and L. Scorrano, "A small lightweight wideband printed dipole for UAV applications," in Proceedings of the 2014 IEEE Antennas and Propagation Society International Symposium IEEE, Memphis, TN, USA, July 2014.

[3] N. M. Boev, "Design and implementation antenna for small UAV," in Proceedings of the 2011 International Siberian Conference on Control and Communications (SIBCON), pp. 152-154, Krasnoyarsk, Russia, September 2011.

[4] J. Choi and J. Kim, "Analysis of optimum antenna placement considering interference between airborne antennas mounted 
on UAV," Journal of the Institute of Electronics and Information Engineers, vol. 52, no. 6, pp. 32-40, 2015.

[5] J.-M. Fernandez Gonzalez, P. Padilla, J. F. Valenzuela-Valdes, J.-L. Padilla, and M. Sierra-Perez, "An embedded lightweight folded printed quadrifilar helix antenna: UAV telemetry and remote control systems," IEEE Antennas and Propagation Magazine, vol. 59, no. 3, pp. 69-76, 2017.

[6] Y. Zeng, R. Zhang, and T. J. Lim, "Wireless communications with unmanned aerial vehicles:Opportunities and challenges," IEEE Communications Magazine, vol. 54, no. 5, pp. 35-42, 2016.

[7] M. Ameen and R. Chaudhary, "Metamaterial based circularly polarized antenna employing ENG-TL with enhanced bandwidth for WLAN applications," IET Electronics Letters, vol. 54, no. 20, pp. 1152-1154, 2018.

[8] M. Ameen, O. Ahmad, and R. K. Chaudhary, "Wideband circularly-polarised high-gain diversity antenna loaded with metasurface reflector for small satellite applications," Electronics Letters, vol. 55, no. 15, pp. 829-831, 2019.

[9] C. Raghvendra and A. Mohammad, "Metamaterial based circularly polarized antenna employing ENG-TL with enhanced bandwidth for WLAN applications," Electronics Letters, vol. 54, no. 20, pp. 1152-1154, 2018.

[10] C. Li, X. Zhu, P. Liu, and C. Yu, "Novel wideband omnidirectional monopole antenna for indoor communication environment," in Proceedings of the 12th European Conference on Antennas and Propagation, pp. 1-3, London, UK, April 2018.

[11] K.-L. Wong, T.-C. Tseng, F.-R. Hsiao, and T.-W. Chiu, "Highgain omnidirectional printed collinear antenna," Microwave and Optical Technology Letters, vol. 44, no. 4, pp. 348-351, 2005.

[12] B. Yang, H. Huang, B. Feng, and L. Deng, "An omni-directional antenna with high gain for WiFi-6 applications," in Proceedings of the 2020 IEEE 3rd International Conference on Electronic Information and Communication Technology (ICEICT), pp. 711-713, Shenzhen, China, November 2020.

[13] L. Akhoondzadeh-Asl, J. Hill, J. Laurin, and M. Riel, "Novel low profile wideband monopole antenna for avionics applications," IEEE Transactions on Antennas and Propagation, vol. 61, no. 11, pp. 5766-5770, 2013.

[14] H. Bukhari and K. Sarabandi, "Miniaturized omnidirectional horizontally polarized antenna," IEEE Transactions on Antennas and Propagation, vol. 63, no. 10, pp. 4280-4285, 2015.

[15] M. Nosrati, A. Jafargholi, R. Pazoki, and N. Tavassolian, "Broadband slotted blade dipole antenna for airborne UAV applications," IEEE Transactions on Antennas and Propagation, vol. 66, no. 8, pp. 3857-3864, 2018.

[16] X. Qing and Z. N. Chen, "Horizontally polarized omnidirectional segmented loop antenna," in Proceedings of the 2012 6th European Conference on Antenna and Propagation (EUCAP), pp. 2904-2907, Prague, Czech Republic, March 2012.

[17] D. Kang and J. Choi, "Compact segmented loop antenna for UAV applications," in Proceedings of the 2017 International Symposium on Antennas and Propagation (ISAP), pp. 1-2, Phuket, Thailand, November 2017.

[18] S. Weiyu, C. Guohu, and Z. Guangqiu, "Dual-band conformal meander-line monopole antenna with coupled patch for unmanned aerial vehicle applications," in Proceedings of the 2016 11th International Symposium on Antennas, Propagation and EM Theory (ISAPE), pp. 171-174, Guilin, China, October 2016.
[19] C. Sairam, T. Khumanthem, S. Ahirwar, and S. Singh, "Broadband blade antenna for airborne applications," in Proceedings of the 2011 Annual IEEE India Conference(INDICON), pp. 1-4, Hyderabad, India, December 2011. 